\title{
Application of " Vertical and Horizontal Cross " Method in Blended Teaching
}

\author{
Jing Liang ${ }^{1, \mathrm{a}}$, ShaoJin Dong ${ }^{1, \mathrm{~b}^{*}}$, Xiang $\mathrm{Di}^{2, \mathrm{c}}$, Chen Shi ${ }^{1, \mathrm{~d}}$ \\ ${ }^{1}$ College of Information and Communication National University of Defense Technology, Xi'an, Shaan'xi, China \\ ${ }^{2}$ College of Information and Communication National University of Defense Technology, Wu Han, He bei, China \\ a1628328@qq.com \\ b*361015138@qq.com \\ crobin_dong@126.com \\ dshishen26@163.com
}

\begin{abstract}
In order to promote the high-quality development and connotative development of education in military academies, it is necessary to make full use of the revolutionary changes brought about by network information technology in education and teaching, and take online and offline blended teaching as the main starting point to promote the implementation of student-centered teaching concept, so as to achieve a qualitative leap in classroom teaching and talent training in military academies. By adopting the method of vertical and horizontal cross, the blended teaching is carried out, so as to promote the teaching reform of the data exchange equipment operation course, and form a new comprehensive professional ability training mode of "advanced stratification, learning classification and target classification".
\end{abstract}

Keywords: Vertical and Horizontal Cross, MOOC, blended teaching

\section{“纵横交叉”法在混合式教学中的应用}

梁璟 ${ }^{1,}$ a董绍进 ${ }^{1,}$ b* 底翔 ${ }^{2,}$ c 时晨 ${ }^{1, d}$

\footnotetext{
1 国防科技大学信息通信学院，陕西西安

2 国防科技大学信息通信学院, 湖北 武汉

a1628328@qq.com

b*361015138@qq.com

crobin_dong@126.com

${ }^{d}$ shishen26@163.com
}

\section{摘要}

为了助力军队院校教育高质量发展和内涵式发展, 要充分利用网络信息技术给教育教学带来的革命性变化, 以 线上线下混合式教学为主要抓手, 推动以学员为中心的教学理念落实落地, 实现军队院校课堂教学和人才培养 质量质的飞跃。通过采用纵横交叉的方法, 来进行混合式教学, 从而来推动数据交换设备操作专业课程教学改 革，形成了一种新的 “进阶分层、学习分类、目标分级” 的全程化专业能力培养新模式。

关键词: 纵横交叉慕课混合式教学

\section{1. 前言}

2020 年 11 月 25 日, 习主席在中央军委军事训
练会议上指出：“要强化科技练兵，增强官兵的科技 素养, 加强新装备新力量新领域训练, 发展先进训练 手段和方法，大幅提高训练科技含量 ${ }^{[1]}$ 。”在这样的 背景下, 我们应主动适应信息技术高速发展带来的变 
化, 大力推动在线开放课程建、用、学, 从而推动混 合式教学的发展 ${ }^{[2]}$ 。

无论是在校还是在线教育, 目标都是全面提高官 兵履职能力和职业素养。具体来说, 就是要求我们组 织各类教学活动时, 在传统课堂教学的基础上, 通过 线上线下混合式教学将官兵成长成才需要同军队建 设发展紧密结合, 普及开展有组织有计划的自主学习, 逐步实现全员、全时、全域的在岗继续教育, 有效解 决广大官兵日益增长的学习需求与学习资源不充分 之间的矛盾。通过线上线下混合式教学 ${ }^{\left[{ }^{3}\right]}$ 使军队人员 得到与军事变革、岗位要求和职业发展相适应的教育 培训, 知识结构及时更新, 履职能力和职业素养全面 提高, 为实现强军目标、建设世界一流军队提供有力 人才保障。

线上教学并非是单纯的在线视频教学, 线下授课 也不再是单纯的课堂讲授和装备训练 ${ }^{[4]}$ 。所以混合式 教学首先要瞄准提高官兵岗位履职核心能力和职业 素养最关键、最急需的知识, 综合运用多种教育技术 手段, 多维度呈现知识和技能, 使受众能够自由选择 最适合自己的方式, 通过自学和教学相结合等多种方 式, 最终达成教育训练目标。与此同时在进行课程内 容选择、教学方法手段、教学环节设计等全过程都要 运用科学的教育教学理论作指导, 避免一成不变低效 率, 或者是盲目摸索走弯路。

如何进行有效的线上线下混合式教学? 通过按 照课程特点分析、教学模式设计、设定学习效果、知 识点梳理归纳、基于问题的学习、多维评价机制、教 学反馈反思的总过程, 确定了利用 “纵横交叉” 的研 究方法来进行混合式教学的分析探索和实践尝试。

“纵横交叉”研究方法需要针对不同类别的知识 点分别采用不同的混合式教学方法, 下面分别介绍。

\section{2. 混合式教学的四个层次}

通过阅读及梳理大量相关文献, 将混合式教学分 为以下四个层次的混合。

\section{1. 第一个层次一一线上与线下的混合}

即将在线教学资源引入传统教室学习。通常理论 类课程适合这个层次的教学模式。主要原因在于, 学 员入校时普遍基础不足、学习能力和学习积极性不高, 需要强有力的监督机制, 才能逐步提升专业基本素质 和自学能力。对于理论类的计算机网络、信息安全技 术两门课程, 慕课平台有大量国家级精品课程可供参 考, 课程的建设和维护质量都很高, 可充分利用这些 慕课和微课 ${ }^{[5]}$ 资源作为课前预习、课后复习和自主深 入学习的材料。

\section{2. 第二个层次一一基于学习目标的混合}

即在 “混合” 策略的设计上以 “达成学习目标” 为最终目标, 混合的学习内容和方式更为广泛。基于 目标的混合式学习既可能都是传统方式的, 例如: 课 堂培训与读书以及讨论会相结合的混合学习, 又可能 都是在线方式的混合, 例如: 通过网络虚拟教室学习 与 BBS 讨论相结合的在线学习等等。因此, 该层次的 混合式学习又被称为 “整合式学习”。理论类课程的 少部分知识和操作类课程主要处于这个层次, 其主要 原因是此时学员已经通过理论类课程掌握相应的专 业基础知识和基本的自学方法, 也通过前置课程的小 组讨论和小组实验等形式建立了较为稳定的相互协 作交流关系。

\section{3. 第三个层次一一 “学” 与 “习” 的混合}

学习, 是指通过阅读、听讲、思考、研究、实践 等途径获得知识和技能的过程。在我们平时的教学过 程中, 通常将学习笼统的作为一个整体去看待。而在 实际教学过程中, 学和习是各自独立又密不可分的两 个部分, 通过将学习过程的学与习进行区分, 可以更 好的了解教学组织中学及习的目标达成情况, 也可以 更有针对性的组织教学。

“学” 与 “习” 的混合才是混合式学习的真正内 涵。通过 “习” 将学习的内容应用到实践中去, 这是 学习更高层次的目的。通常情况下, 我们将 “学” 等 同于学习, 而实际情况将 “习” 完全遗漏掉了, 绝大 多数的面授或在线学习都只是 “学” 而已, 并不是真 正意义的学习。实际上, 设计 “学” 与 “习” 的混合 才是最有效的混合式学习。而这种混合式学习在设计 上其实又是最核心的。操作类课程的部分内容和综合 训练类课程都处于这个层次。这个层次的难点在于, 知识的 “习”所用的时间可能远远多过 “学” 的时间, 才能达成课程学习目标。这就要求教员要综合使用多 种方法手段, 调动学员利用课余时间进行 “习” 的积 极性和主动性。

为了更方便直观的区分学与习的不同类型，下面 以广域网接入技术为例通过表 1 进行说明。

表 1 “学”与 “习” 分类示例

\begin{tabular}{|c|c|c|}
\hline 内容 & \multicolumn{2}{|c|}{ 学与习分类 } \\
\hline PPP、静态路由、TELNET 综 & 高级运用 & \\
\hline 合训练 & 习 \\
\hline PPP 配置单点达标 & 基础掌握 & \\
\hline PPP 配置流程及命令 & 技能理论 & \\
\cline { 2 - 2 } & & \multirow{2}{*}{ 学 } \\
\hline PPP 协议理论 & 专项理论 & \\
\hline 广域网接入技术 & 基础理论 & \\
\hline
\end{tabular}

\section{4. 第四个层次一一学习与工作的混合}

学习与工作相结合的混合式学习又被称为 “嵌入 式” 的学习或 “行动学习”, 与其说是一种学习方法, 不如说是一种学习境界, 也可以称为深度学习 ${ }^{[6]}$ 。从 
某种意义上来说, 工作本身就是学习。通常综合训练 类课程的部分内容处于这个层次。当学员走上工作岗 位以后就一直处于这个层次, 这又要求我们为处于工 作状态的学员提供 “学” 的途经, 也就是目前正在大 力发展的军事职业教育。

\section{3. 知识点分类}

不同课程有不同的总体培养目标, 而一门课程内 部不同知识和技能点也有不同的特点, 比如偏理论、 偏操作或是纯理论、纯操作等等。通过对数据交换设 备操作和网络安防两个专业的相关网络类课程进行 分析, 归类, 根据课程定位, 以及理论内容和实践内 容的比例, 我们将课程大致分为三类: 理论类、操作

表 2 知识单元和知识点的分类
类以及综合运用类。

《路由交换设备操作》课程总体上属于操作类, 但具体到知识和技能点上则可能属于理论类、操作类 或综合运用类等。

这就要求学习的双方都要采取适当的学习策略 来分别传授和掌握知识点。课程开始前, 授课者需要 对课程知识点进行梳理归纳, 针对不同混合式教学层 次的知识点, 分别设计不同的混合式教学方法策略。 以《路由交换设备配置》这门课为例, 将其共分解为 4 个模块, 每个模块细分为若干个知识单元和知识点 并对其分类。表 2 列出了课程第一个教学模块的知识 单元和知识点的分类情况。

\begin{tabular}{|c|c|c|c|c|c|}
\hline 序 & $\begin{array}{l}\text { 教学 } \\
\text { 模块 }\end{array}$ & $\begin{array}{l}\text { 知识 } \\
\text { 单元 }\end{array}$ & 知识点 & 分类 & 目标 \\
\hline \multirow{2}{*}{1} & \multirow{8}{*}{$\begin{array}{l}\text { 网络设备 } \\
\text { 基本配置 }\end{array}$} & \multirow{2}{*}{ 基本原理 } & 路由器工作原理 & 理论类 & 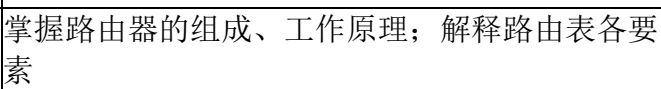 \\
\hline & & & 路由器接口和互连 & 理论类 & $\begin{array}{l}\text { 辨认路由器的常用接口; } \\
\text { 阐述路由器接口常见连接方式 }\end{array}$ \\
\hline \multirow[b]{2}{*}{2} & & \multirow[b]{2}{*}{ 登录与配置 } & 路由器登录方法 & 理论类 & 了解访问路由交换设备的各种方法 \\
\hline & & & 路由器登录操作 & 操作类 & $\begin{array}{l}\text { 会通过 Console 端口和 Telnet 访问路由交换设 } \\
\text { 备; 掌握常用维护配置命令的操作使用 }\end{array}$ \\
\hline \multirow{4}{*}{3} & & \multirow{4}{*}{ 广域网接入技 } & 广域网接入技术 & 理论类 & 理解广域网常见的链路层封装 \\
\hline & & & 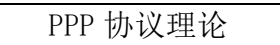 & 理论类 & 理解 PPP 协议原理和工作过程 \\
\hline & & & $\begin{array}{l}\text { PPP 认证配置 } \\
\text { 流程及命令 }\end{array}$ & 操作类 & 掌握 PPP 认证的配置流程和命令 \\
\hline & & & PPP 的组网运用 & 综合运用类 & 会运用 PPP 协议构建广域互联网络 \\
\hline
\end{tabular}

\section{4. 纵横交叉分析法总体思路}

以知识点类型为纵轴, 以混合的层次为横轴, 在 纵向分解和横向分析的基础上, 将知识点和这四个层 次进行纵横交叉, 根据交叉的结果, 便可以得到各知 识点适宜采用的混合式教学方法策略, 从而交汇形成 课程设置和教学实施的教学改革内容。图 1 以常用知 识点为例, 表示了使用纵横交叉方法对知识点进行分 类的过程, 然后将分类后的知识点按照表 3 的混合式 教学组织方法制定对应的教学实施计划。

在进行混合时, 整个学习过程可以被规划为三个 阶段。首先通过将线上教学资源引入传统教学, 并进 行课程资源的归类和课程内容重构, 明确需要 “学”
的教学内容, 规划 “习” 的训练部分, 使其符合学生 的岗位能力; 然后对 “学”与 “习” 这两部分内容又 分别按照知识和能力的进阶规律进行细分; 接着根据 细分后的教学内容和学员学习能力差异采用多层混 合式教学方法, 以 “达成学习目标” 为目的, 调动学 员 “习” 的积极性和主动性, 通过 “习” 将学习的内 容应用到实践中去; 当然还要为学员提供持续 “学” 及 “习” 的途经和方法, 从而提高学员的履职能力和 职业素养。

由于传统的基于工作任务的课程开发模式只重 视阶段的连贯性, 忽略了课程设计内在的有机性, 因 此造成最终开发的课程整体性不够、系统性不足。而 横纵结合的研究方法, 能够有效避免这种现象的发生。 


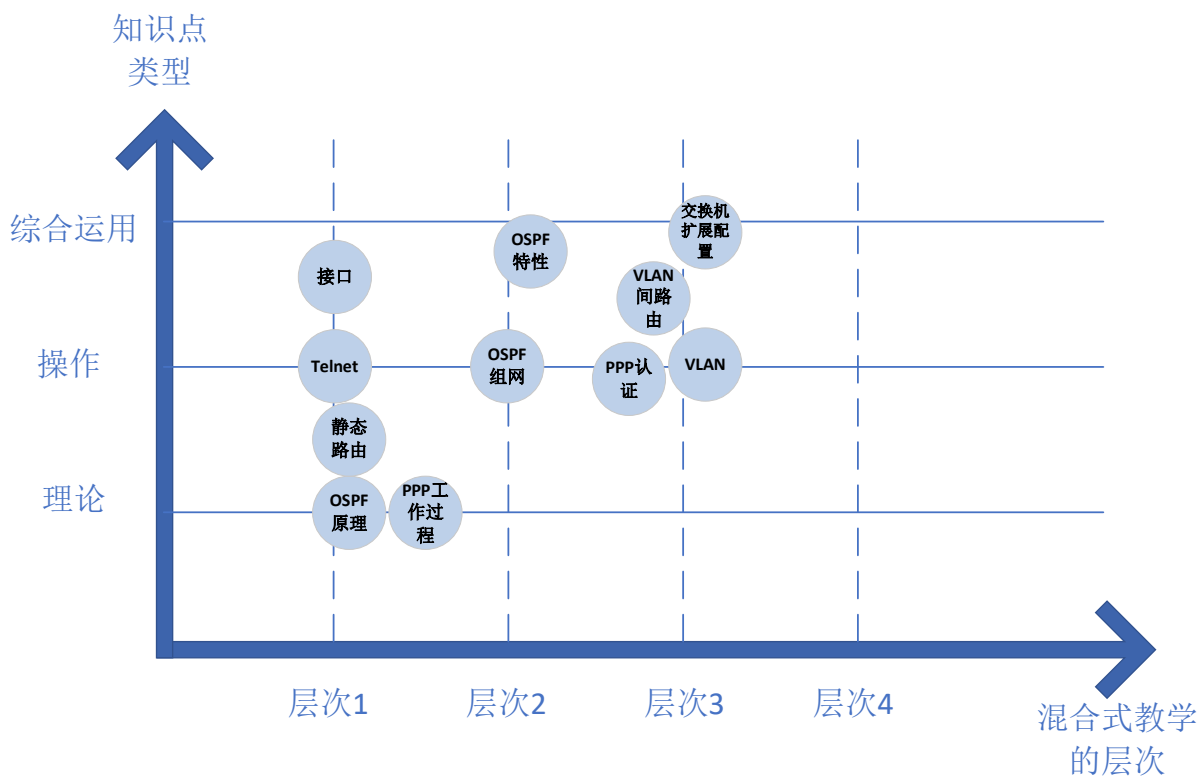

图 1 “纵横交叉”方法示意图

表 3 “纵横交叉” 方法指导下的混合式教学组织方法

\begin{tabular}{|c|c|c|c|c|}
\hline & \multicolumn{3}{|c|}{ 混合式教学组织 } & 在岗学习 \\
\hline 综合运用类 & $\begin{array}{c}\text { 线上慕课自学 } \\
\text { 精讲理论 } \\
\text { 线下训练 }\end{array}$ & $\begin{array}{c}\text { 线上慕课自学 } \\
\text { 精讲理论 } \\
\text { 小教员演示 } \\
\text { 线下训练 }\end{array}$ & $\begin{array}{c}\text { 线上慕课自学 } \\
\text { 精讲理论 } \\
\text { 讨论辩论 } \\
\text { 小教员演示 } \\
\text { 线下训练 }\end{array}$ & $\begin{array}{l}\text { 线上慕课自学 } \\
\text { 线上模拟训练 } \\
\text { 线上讨论交流 }\end{array}$ \\
\hline 操作类 & $\begin{array}{c}\text { 线上慕课自学 } \\
\text { 线下训练 }\end{array}$ & $\begin{array}{c}\text { 线上慕课自学 } \\
\text { 线上模拟训练 } \\
\text { 线下训练 }\end{array}$ & $\begin{array}{c}\text { 线上慕课自学 } \\
\text { 线上模拟训练 } \\
\text { 小教员演示 } \\
\text { 线下训练 }\end{array}$ & $\begin{array}{l}\text { 线上慕课自学 } \\
\text { 线上模拟训练 } \\
\text { 线上讨论交流 }\end{array}$ \\
\hline 理论类 & $\begin{array}{c}\text { 线上慕课自学 } \\
\text { 精讲理论 }\end{array}$ & $\begin{array}{c}\text { 线上慕课自学 } \\
\text { 精讲理论 } \\
\text { 小组合作学习 }\end{array}$ & $\begin{array}{c}\text { 线上慕课自学 } \\
\text { 精讲理论 } \\
\text { 小组合作学习 } \\
\text { 讨论辩论 }\end{array}$ & $\begin{array}{l}\text { 线上慕课自学 } \\
\text { 线上讨论交流 }\end{array}$ \\
\hline & 层次 1 & 层次 2 & 层次 3 & 层次 4 \\
\hline
\end{tabular}

\section{5. “纵横交叉” 法在混合式教学中的应用}

运用 “纵横交叉” 分解方法，设置课程前、课程
中、课程后三环节，采取目标分级、全程考核评价方 式, 结合教学目标、方式方法、学员学习三个维度, 探索出了一条 “进阶分层、学习分类、目标分级” 的 全程化专业能力培养新模式。总体设计如图 2 所示。 


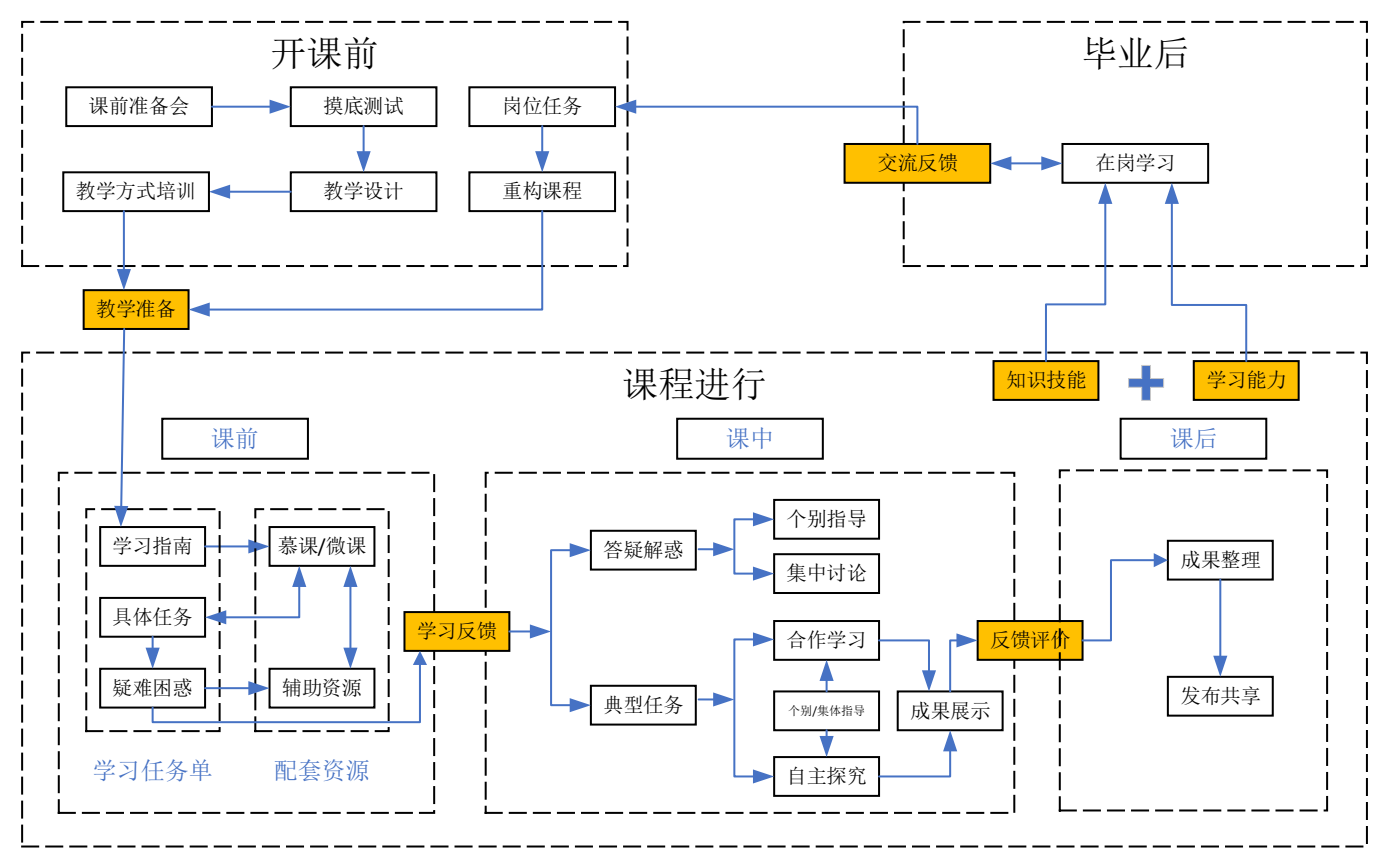

图 2 混合式教学实施的基本流程图

为了能更清楚的介绍该混合式教学的实施过程, 下面将分别从三个维度具体介绍。

\section{1. 目标维度}

教学目标的设定主要在课程开课前完成, 在课中 和课后学员可根据学习难度、进度和学习效果评价情 况反馈，适当调整教学策略及方法。

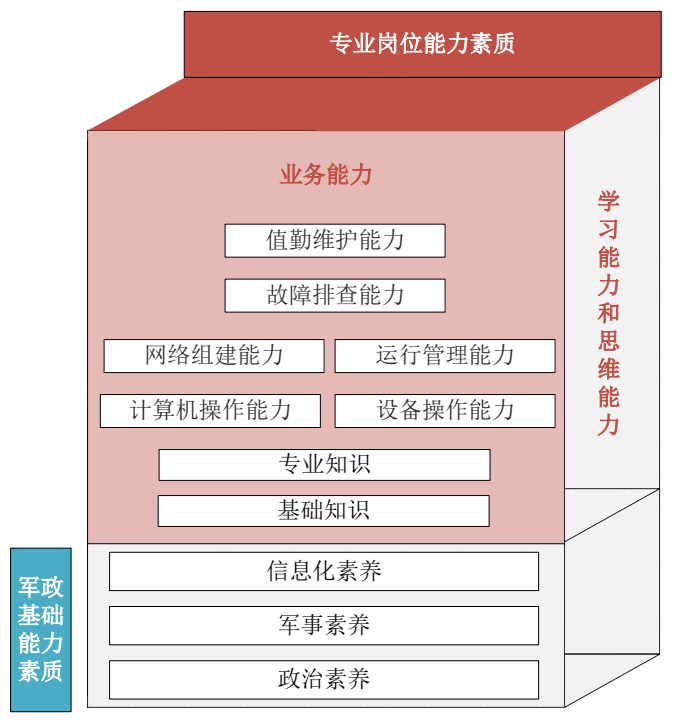

图 3 专业能力模型图

根据该层次和专业的培训方案, 学员在完成培训 后的知识、能力和素质方面的要求主要包括以下方面: (1) 知识上, 了解数据交换设备操作专业理论知识, 熟悉数据交换设备技术性能和值勤管理规定; (2) 能 力上, 掌握必需的计算机基础知识, 具备初步的计算 机操作能力; 掌握路由器交换机的配置命令和重要业 务的配置方法, 具备一定的数据交换设备配置能力;
掌握网络服务器配置方法, 具备一定的网络服务管理 能力; (3) 素质上, 具备持续学习意识和初步的创新 精神，能够胜任数据交换设备值机员岗位。

将其绘制成专业能力模型如图 3 所示。

\section{2. 组织维度}

将该课程的整体教学过程分为课前 (分析、设计、 组织)、课中 (实施) 和课后 (评价) 等三个环节五个 步骤, 以保证高效地进行该混合式教学课程的组织与 实施, 如图 4 所示。

\subsection{1. 教学分析}

主要从教学对象、教学内容和教学环境三个方面 进行分析。

在教学目标维度中, 课题组已经完成了教学对象 的知识基础和学习能力情况分析估计, 总结出了本班 次学员的共性和个性。

为了更好地实施混合式教学, 课题组将培训方案 中规定的教学训练内容, 按照对知识、技能/能力和 素质的不同培养目标, 按照难易程度和是否适合混合 式教学等因素进行分类, 梳理归纳成知识技能图谱。

再根据知识技能教与学的教学环境需求, 创设改 进网络和课堂两个环境, 提出混合式教学资源需求, 并找准混合式教学结合点。混合式教学环境主要包括 网络数字化学习环境和课堂教学活动环境。网络数字 化学习环境包括网络硬件环境的配置、网络学习平台 及其可用资源的建设、学员可用网络学习设备的环境 配置等软硬件环境资源; 课堂教学活动环境主要包括 带有多媒体教讲环境的实验室等。 


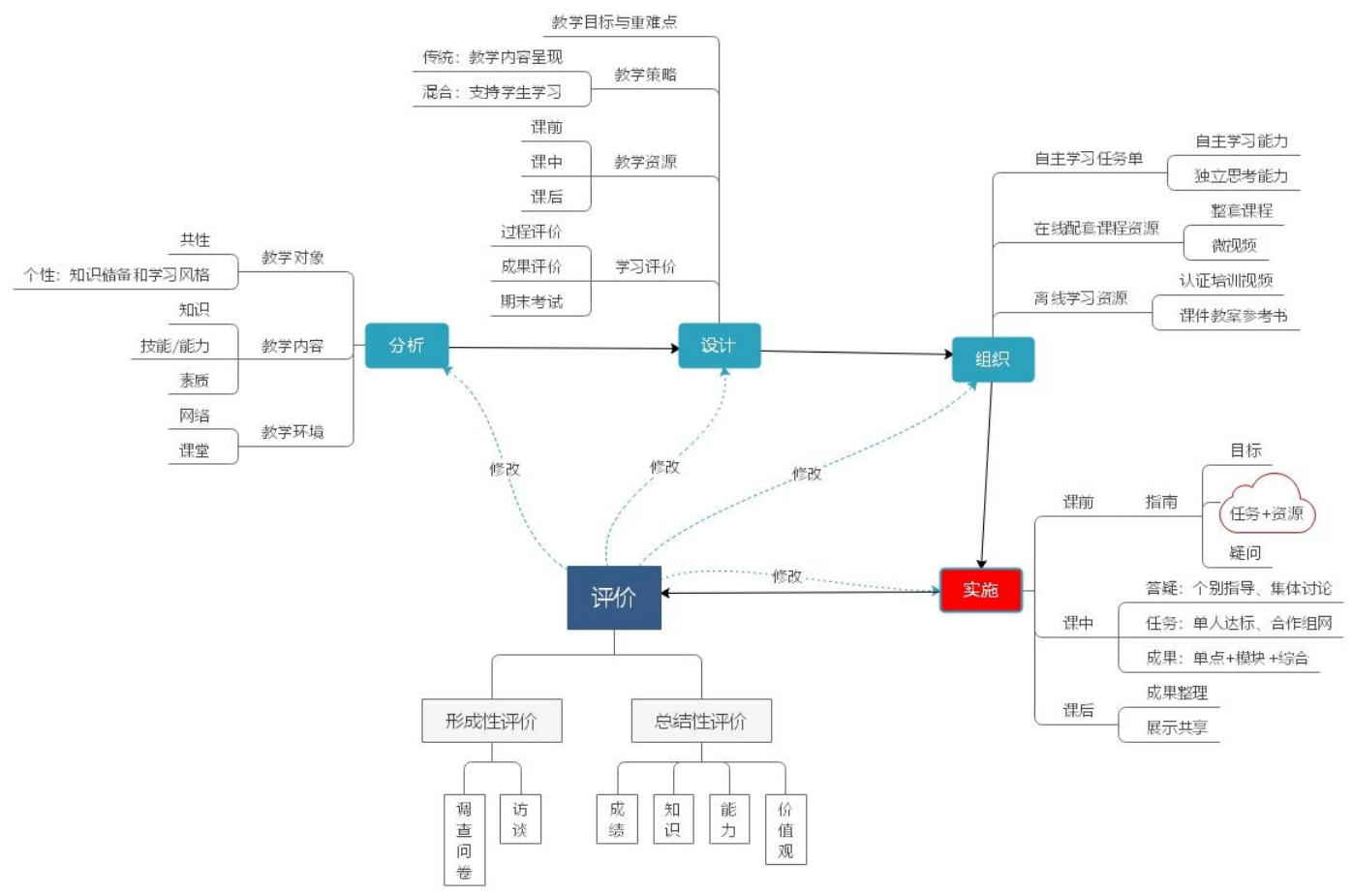

图 4 混合式教学课程的组织与实施示意图

\subsection{2. 教学设计}

基于前述分析结果, 课题组认为, 设计阶段应主 要从教学目标与重难点、教学策略、教学资源、学习 评价等四个方面进行设计。

教学目标与重难点设计是混合式教学设计的灵 魂所在, 对后续阶段起着统领作用。结合对教学对象 和教学内容的分析, 可从知识、能力、情感态度价值 观等三方面对教学目标进行分类阐述, 并由此确定教 员教学和学员学习的重难点, 使得混合式教学活动的 开展有的放矢。

教学媒体选择和教学策略设计是为了实现教学 目标, 而选择并确定信息传递的通道以及师生开展教 与学活动的组织方式。混合式教学与传统教学的核心 区别之一, 就在于教学媒体选择和教学策略设计的不 同一一传统教学中教学媒体的选择主要考虑如何更 加有助于教员教学内容的呈现, 而混合式教学中教学 媒体的选择更多地偏重于哪些媒体形式能够更好地 支持学员的学习; 为了更好地激发学员主动学习的动 机、促进学员的深度学习, 混合式教学策略设计需要 考虑教学组织形式, 如采用课堂环境的小组合作学习、 自主探究学习、讨论辩论式学习以及课下真实任务驱 动的研究性学习等, 以真正将 “学员为主体” 落实在 具体的教学策略设计过程中。

教学过程与教学资源设计是在教学策略确定之 后, 围绕学习活动而开展的具体教学过程和资源的设 计。在混合式教学中, 教学过程设计不再仅仅局限于 传统的课堂教学这一个环节, 而是围绕学员课前、课
中和课后三个阶段学习活动而进行整合的设计, 包括 课前自主学习任务单的设计和网络学习资源的设计、 课堂教学与研讨活动的策划与设计、课下研究性学习 活动的设计等环节。

学习评价设计是混合式教学设计的最后一个环 节, 通过学习过程的评价 (如学习平台的用户日志和 在线测验完成情况)、研究性学习成果评价 (如组内 自评与互评、组间互评、师生互评) 和课程的期末考 试等三个方面进行学员学习效果的评定。

\subsection{3. 资源组织}

立足于分析、设计两个阶段，开发阶段重在选择 合适的教材资源，同时制作、开发和收集整理各种辅 助学习资源, 以生成具体的教学单元内容。在开发阶 段, 课前需要完成与学员自主学习相关的配套资源的 开发, 主要包括自主学习任务单的制作和以慕课/微 视频为核心的在线配套课程资源的建设。需要重点强 调的是, 自主学习任务单是引导学员利用配套学习资 源开展学习活动、完成学习任务、实现教学目标的学 习支架, 因此, 以慕课/微视频为核心的在线配套课 程资源的开发活动应该基于自主学习任务单中的学 习任务设计; 同时, 要明确自主学习任务单中任务设 计的核心是将教学内容的重难点转换为面向真实情 境的问题解决, 培养学员的自主学习和独立思考能力。

\section{2. 4. 教学实施}

在具体实施过程中, 区分知识基础和学习能力, 将学员按强弱搭配分为若干学习小组, 组内帮扶、组 
间评比, 兼顾知识能力掌握情况和进步情况, 以提升 所有学员的学习积极性。

\section{2.5. 评价反馈}

混合式教学设计的价值体现依赖于评价阶段, 而 评价主要包含形成性评价和总结性评价一一形成性 评价贯穿于混合式教学设计的各个阶段, 通过调查问 卷、访谈等方式收集数据, 并在后期阶段中对教学设 计方案予以不断完善; 总结性评估则在教学实施完成 后进行, 主要就学员的学习成效、知识掌握、能力养 成、价值观完善等进行全面的考察和评鉴，并据此修 正教学设计的五个步骤, 通过迭代式的循环, 形成混 合式教学设计的最佳实践。

\section{3. 学习维度}

学习维度是在考虑每个学员差异化的基础上, 从 课程开课前、课程实施中以及知识技能向素质提升三 个方面设计完成的。

在课程开课前, 先对学员不同的个体学习基础, 进行前期热身辅导, 具体实施过程如下。

通过入学前岗位和学历情况分析、摸底测试、前 置课程学习考核情况和问卷调查了解个体学习基础, 然后按照强弱搭配的原则组成多个学习小组, 并进行 适当的混合式学习培训。

在课程进行过程中, 针对某个具体知识点进行学 习的流程如下。

学员首先受领自主学习任务单, 将自主学习过程 中发现的问题, 通过小组帮扶和在线答疑等形式进行 讨论交流; 对于尚未解决的重难点问题, 通过课堂精 讲和针对性训练, 引导学员自主分析和解决问题, 并 产生实验报告、个人小结等学习成果; 然后通过单点 达标考核检查、评价和总结该知识点的学习情况。在 学员查缺补漏的同时, 受领新知识点的自主学习任务 单。

为了更好的将知识和技能内化为能力素质, 在每 个教学模块结束时以小组为单位组织模块综合训练 及测评, 并进行简单故障检测与排除; 在完成所有知 识点学习后, 以小组为单位完成基于实战背景的综合 组网, 全面提升学员学员的规划设计、业务配置和管 理维护能力。

\section{6. 结语}

本文针对线上线下混合式教学组织形式及内容 有待优化的问题进行了研究, 设计了 “纵横交叉” 的 研究方法来对混合式教学进行组织, 形成了一种 “进 阶分层、学习分类、目标分级” 的数据交换设备操作 专业人才培养质量提升模式。通过采用这种方法进行 混合教学, 可以更好实施教学组织过程、丰富设备操 作与维护等实践类教学的活动形式、促进学员创新实
践能力提升, 取得较好的教学效果。

\section{项目基金}

本文为国防科技大学信息通信学院 2019 年度教 学研究重点课题——基于慕课的 “路由交换设备配 置”课程混合式教学探索与研究的阶段性成果之一。 (课题编号: JY19A002)

本文为国防科技大学信息通信学院金课建设阶 段性成果之一。

\section{REFERENCES}

[1] Comprehensively strengthen practical military training and comprehensively improve training level and winning ability [n]. PLA Daily, 2020-11-26 (01).

[2] Yang Weidong, Dong Xiaoyu. Dilemma and optimization path of network curriculum construction in Colleges and universities [J]. China higher education, 2019 (10): 48-50.

[3] Cooney M. H, Gupton P, O' Laughlin M. Blurring the lines of play and work to create blended classroom learning experiences[J]. Early Childhood Education Journal, 2000(3) : 165-171.

[4] Zheng Jing. Investigation and Analysis on the current situation of blended teaching in domestic colleges and universities [J]. Heilongjiang Higher Education Research, 2018 (12): 44-47

[5] Wang Shengqing, Feng Xuesong. Design and practice of Blended teaching Based on Mu class [J]. Modern educational technology, 2017 (11): 71-77

[6] Tan Shuang. Construction of "blended teaching" mode in Colleges and universities pointing to deep learning [J]. China higher education, 2019 (6): 5153. 